\title{
Mitteilungen der Österreichischen Gesellschaft für Klinische Pathologie und Molekularpathologie
}

Pathologe $2021 \cdot 42: 143-145$

https://doi.org/10.1007/s00292-020-00895-5

C) Springer Medizin Verlag $\mathrm{GmbH}$, ein Teil von Springer Nature 2021

\section{Verantwortlicher Redakteur}

Dr. Nicolas Binder (V.i. S. d. P.)

Pathologisch-bakteriologisches Institut

SMZ Süd

Kundratstraße 3

1100 Wien

nicolas.binder@wienkav.at
Österreichische Gesellschaft für Pathologie / Österreichische Abteilung der IAP (ÖGPath/IAP Austria)

Institut für Klinische Pathologie und Molekularpathologie Landesklinikum Mistelbach-Gänserndorf Liechtensteinstraße 67

2130 Mistelbach

Tel.: +43(0)15366326

Fax.: +43(0)15366361

office@oegpath.at

\section{Rückblick 2020}

\section{Frühjahrstagung der ÖGPath/IAP Austria \\ - Gemeinsame Fortbildungstagung \\ für Biomedizinische Analytikerlnnen und ÄrztInnen}

\section{1.-12. September, Wien}

Die Frühjahrstagung der ÖGPath/IAP Austria stand organisatorisch gänzlich im Zeichen der COVID 19-Pandemie. Ursprünglich für März 2020 geplant wurde sie letztlich auf September verschoben und konnte dank eines minutiös geplanten Präventionskonzeptes als Präsenzveranstaltung im TechGate Wien abgehalten werden.

Inhaltlich stand die Tagung im Zeichen der Mammapathologie, insbesondere in Bezug auf die neue WHO-Klassifikation.

Am Freitag startete das Programm parallell mit dem traditionellen Treffen der AG Pulmopathologie und einem Block über

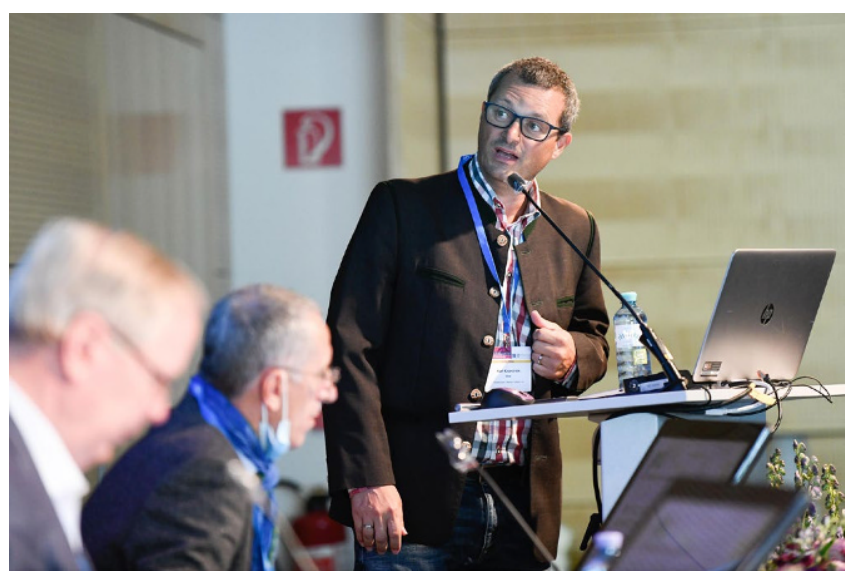

Abb. $1 \Delta$ Dr. Kashofer über Akkreditierung die praktische Umsetzung der Akkreditierung nach ISO 15189 (- Abb. 1). Es folgte ein Gespräch zwischen Pathologie (Peter Regitnig, Graz) und Klinik (Rupert Bartsch, Wien) über Biomarker beim Mammakarzinom, das den großen Themenblock der Mammapathologie einleitete (- Abb. 2).

Der erste Block behandelte den Themenkomplex der Mammapathologie in der täglichen Praxis. Doris Kriegl (Graz II) Milena Öttl (Graz II), und Sigurd Lax (Graz/Linz) referierten über standardisierte Mammadiagnostik während Prof. Regitnig über die Anwendung von Machine learning bei der Befundung von Sentinel Lymphknoten sprach.

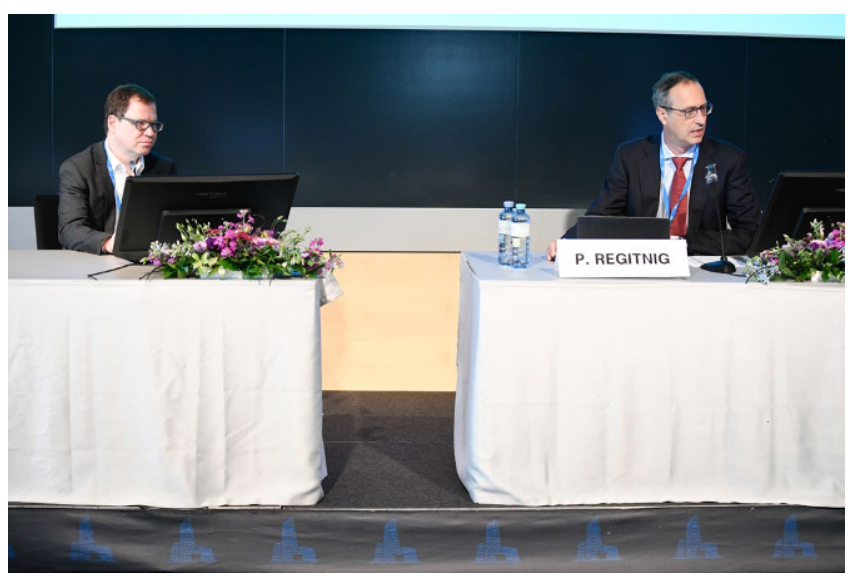

Abb. 2 A Prof. Bartsch und Prof. Regitnig

Am Nachmittag beschäftigten sich die Vorträge zunächst mit der neoadjuvanten Therapie des Mammakarzinoms aus Sicht der Pathologie (Zsuzsanna Bago-Horvath, Wien) (• Abb. 3), der Chirurgie (Florian Fitzal, Wien), der Radiologie (Mag. Dr. Maria Bernathova, Wien), der Klinischen Onkologie (Rupert Bartsch) und der Radioonkologie (Daniela Kauer-Dorner).

Den Abschluss bildete ein Block über die neue WHO-Klassifikation mit Vorträgen von Emad A. Rakha (Nottingham) und Anna Sapino (Turin).

Der Samstag setzte sich fort mit weiteren wichtigen und interessanten Themen der Mammapathologie.

Das Thema PIK3CA Mutationen beim Mammakarzinom wurde von Leonhard Müllauer, AKH Wien besprochen - „Wieso - Wie - Wann und Woraus“. Im nächsten Themenblock zur aktuellen WHO-Tumorklassi- fikation der Mamma waren unsere internationalen Referenten Paul van Diest (NL) und Emad A. Rakha (GB) per Skype zugeschaltet. $\mathrm{Zu}$ weiteren aktuellen Themen mit Bezug zur Mamma waren aufgrund der COVID-Situation weitere internationale Referenten virtuell zugegen. Wir bedanken uns bei Roberto Salgado (BE), Zsuzsanna Varga (CH) und Paul van Diest (NL) für ihre interessanten Beiträge per Skype $\mathrm{zu}$ „Combined TILS-PDL 1 in Breast Cancer“, „Standardized and synoptic reporting of Breast Cancer" sowie „Biomarkers an gene expression assays".

Nach der Mittagspause folgten unsere Teilnehmer dem interessanten Schnittseminar. Sigurd Lax, Peter Regitnig, Anna Sapino und Zsuzsanna Varga haben ihre Fälle vorab zur Verfügung gestellt. Die Präsentation der interessanten Fälle wurde abgerundet durch ein „Digivoting“, das von 


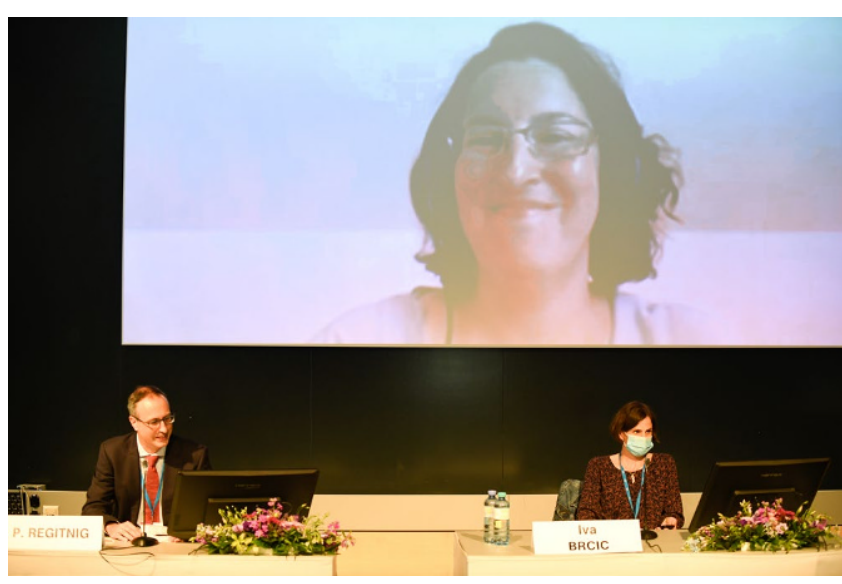

Abb. $3 \Delta$ Prof. Bago-Horvath per Livestream zugeschaltet

fast allen Teilnehmern und Teilnehmerinnen genutzt wurde.

In diesem Sinne wollen wir uns beim Wissenschaftlichen Komitee, bei allen Referenten und Referentinnen für das Update Lungenpathologie sowie die großartige Umsetzung des Themas Mammapathologie bedanken.

\title{
6. Patholauf der ÖGPath/IAP Austria
}

Insgesamt 14 Teilnehmerinnen und Teilnehmer sind zeitig aufgestanden, um am Samstag, den 12. September 2020, am 6. Patholauf der ÖGPath/IAP Austria

teilzunehmen. Bestens gestimmt legten die Läuferinnen und Läufer eine Strecke von $5 \mathrm{~km}$ in schönster Morgenstimmung im Donaupark zurück. (• Abb. 4)

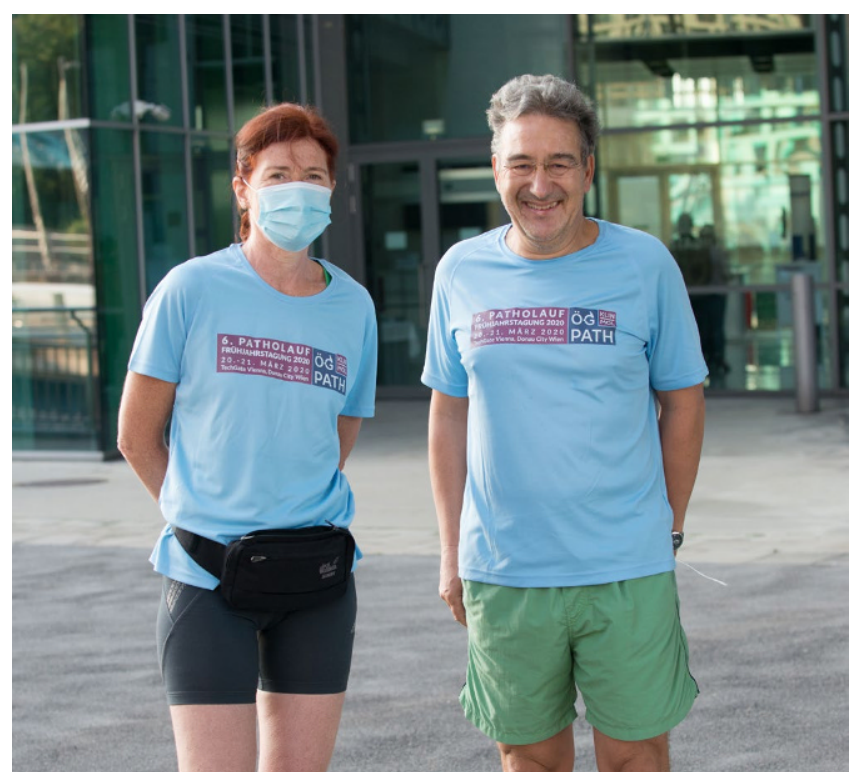

Abb. $4 \Delta$ Der traditionelle Patho-Lauf konnte trotz Pandemie abgehalten werden (Prim. Freibauer und Prim. Hulla)

\section{Pathology Future Academy - Intensivseminar Wissenschaftliches Arbeiten}

\author{
10.0ktober 2020, VIRTUELL
}

Die 2. Auflage des Intensivseminars „Wissenschaftliches Arbeiten" im Rahmen der Veranstaltungsreihe Pathology Future Academy der ÖGPath/IAP Austria hat am Samstag, dem 10. Oktober 2020 virtuell stattgefunden.

Konzentriert haben 30 TeilnehmerInnen den spannenden und interessanten Ausführungen der Referenten gefolgt. Die Form der virtuellen Seminargestaltung hat sich, dank dem erfahrenen Team von der Medizinischen Universität Wien, für diese Veranstaltung bestens bewährt.

Im ersten Teil des Programmes, das Renate Kain, MedUni Wien, zusammengestellt hat, wurden Rahmenbedingungen des Wissenschaftlichen Arbeitens wie Ethik (Martin Brunner, MedUni Wien), die Regeln der Good Scientific Practice inkl. Datenschutzgrundverordnung (Jessica Einzinger, MedUni Wien), Gewebe- und Biobank (Philipp Hofer) berücksichtigt. Andreas Chott hat wertvolle Tipps geliefert, um leidvollen Erfahrungen rund um das Wissenschaftliche Präsentieren geliefert.

Im zweiten, praktisch orientierten Teil der Veranstaltung hat
Renate Kain für uns den Bogen von der Identifikation und Auswahl einer biomedizinisch-ethischen Fragestellung über die Formulierung der Hypothese bis zur Beantwortung der Forschungsfrage gespannt. Ebenso wurden die wesentlichen Themen wie Ausarbeitung eines Projektes inkl. Zeit- und Finanzierungsplan und Fördergeber besprochen. Gerald Höfler (MedUni Graz hat anhand von praktischen Beispielen eingeführt in das Vorbereiten eines Abstracts oder einer Publikation. Abgerundet wurde das Programm durch Sigurd Lax (JKU Linz, Graz), der Wesentliches zum Publizieren aus der Sicht des Editors berichtet hat.

Wir freuen uns sehr, dass die TeilnehmerInnen des Seminars vom Erfahrungsschatz der Referenten profitieren können und auf diese Art in ihrer Motivation, sich kritisch mit der Literatur auseinanderzusetzen bzw. selbst wissenschaftlich aktiv zu werden, Unterstützung finden und bedanken uns bei allen Referentinnen, Referenten und Organisatoren.

\section{Verleihung der ÖGPath- Wissenschaftspreise}

Die ÖGPath/IAP Austria hat im Rahmen ihrer Frühjahrstagung in Wien ihre Wissenschaftspreise und Forschungsförderungen verliehen. Der mit $€ 5000$ dotierte Carl von Rokitansky-Preis wurde an Dipl.Ing. Mag. Monika Oberhuber von der Medizinischen Universität Wien für ihre
Arbeit STAT3-dependent analysis reveals $P D K 4$ as independent predictor of recurrence in prostate cancer publiziert in Molecular Systems Biology verliehen.

Ein Start-up Grant des Forschungsförderungsprogramms der ÖGPath/IAP Austria wurde an Dr. Maja Nackenhorst für ihr 


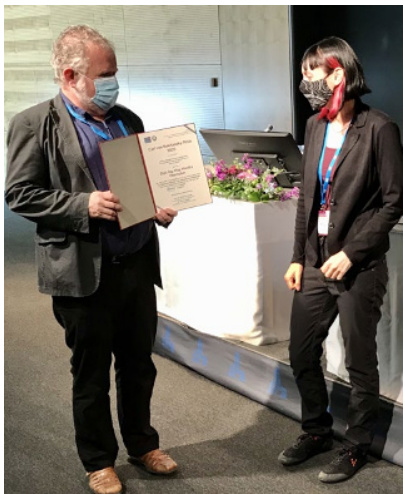

Abb. $5 \Delta$ Verleihung des Rokitansky-Preises

Projekt Evidence of Parvoviridae and SARS-CoV-2 in renal biopsies with tubulointerstitial nephritis of unknown origin and patients of SARS-CoV-2 infection vergeben. Der Grant ist mit max. $€ 10.000$ dotiert.

Die feierliche Verleihung des Preises erfolgte durch den Pastpresident der ÖGPath/IAP Austria und Vorsitzenden des Carl von Rokitansky-Preiskomitees sowie des Auswahlkomitees des Forschungsförderungsprogramms Univ.Prof. Dr. Gerald Höfler. (• Abb. 5)

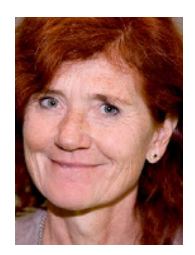

Prim. Dr. Christa Freibauer

Präsidentin

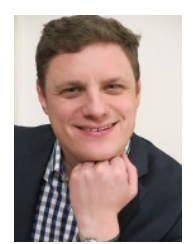

Dr. Nicolas

Binder

Schriftführer

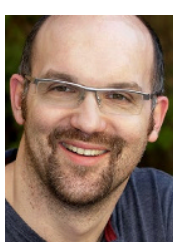

Priv.Doz. DDr. Luka Brcic Internationaler Sekretär

\section{Die ÖGPath bei der MedKarriere-Messe 2020}

Aufgrund der COVID 19-Pandemie konnte die MedKarriere Messe diesmal nur virtuell stattfinden. Die ÖGPath/IAP Austria war dennoch mit einem Stand

\section{Vorankündigungen}

\author{
Mammographie Scree- \\ ningkurs
}

\author{
26.-27.02.2021 \\ VIRTUELL
}

\section{Frühjahrstagung 2021 der ÖGPath/IAP Austria}

9.-10.04.2021

VIRTUELL

\section{Pathology Future Academy - Intensivseminar Mikro- biologie - Modul 1}

\section{8. -30.04 .2021$}

Ried

\section{Pathology Future Academy - Intensivse- minar Klinische Pathologie (verschoben aus 2020)}

\section{2.-04.05.2021}

Seggau 6th Pannonia Congress of Pathology

19.-22.05.2021

Jasnà, Niedere Tatra, Slowakei

Pathology Future Academy - Intensivseminar Mikrobiologie - Modul 2

10.-11.06.2021

Graz

Herbsttagung 2021 der ÖGPath/IAP Austria

16. -18.09 .2020$

Villach

Pathology Future Academy - Intensivseminar Klinische Pathologie

28.-30.11.2021

Seggau

\section{Pathologie.Recht 2022}

Zauchensee und einem Vortrag vertreten, um Medizinstudenten für das Fach Klinische Pathologie und Molekularpathologie zu begeistern. 